Environment Conservation Journal 19 (3) 123-128, 2018

ISSN 0972-3099 (Print) 2278-5124 (Online)

Abstracted and Indexed

\title{
Assessment of ground water quality of Laksar block in district Haridwar, Uttarakhand
}

\author{
Bhutiani R. ${ }^{1}$, Ram Khushi ${ }^{1}$ 凶, Tyagi Varun ${ }^{1}$, Ahamad Faheem ${ }^{1}$ and Kaushik Pankaj ${ }^{2}$
}

Received: 25.06 .2018

Revised: 28.08 .2018

Accepted: 14.10.2018

\begin{abstract}
The present study was aimed to identify the ground water contamination problems in Laksar block of district Haridwar. The ground water samples were collected from 6 different locations of Laksar block. The Physico -chemical parameters were assessed to check either the water is suitable or not for drinking purpose. The Physico-chemical parameters such Temperature, Total hardness (TH), Calcium Hardness (CaH), Acidity, Chloride, Sulphate, pH and Electrical Conductivity (EC) were analysed as per standard methods. In this study values of different parameters were recorded such as acidity (78.6 to $79.4 \mathrm{mg} / \mathrm{l})$, pH (6.36 to7.11), chloride (111.8 to $113.8 \mathrm{mg} / \mathrm{l})$ and total hardness (370.2 to $374.6 \mathrm{mg} / \mathrm{l})$ respectively. This study revealed that water samples may raise concern on the safety of the water for human health may cause the various water borne disease, therefore, the proper hygiene and purification techniques should be recommended.
\end{abstract}

Key words: Assessment, Ground Water, Laksar Block, Haridwar

\section{Introduction}

Water is the important constituent of all things without water life is impossible on the earth (Arya et al., 2011). Water is required to all forms of life and make up 50-97\% of the weight of all plants and animals. About $70 \%$ of the water in India has become polluted due to the discharge of domestic sewage and industrial effluents into natural water source, such as rivers, streams as well as lakes (Sangu and Sharma, 1987). Almost 95\% of rural population living in India depends on ground water for domestic use (Moharir et al., 2002). Ground water is of great important in agriculture for both watering of crops and for irrigation of dry season crops. The term groundwater is used to denote that water which has saturated the pores or aperture of the sub-soil. About $45 \%$ of irrigation water requirement is met from ground water sources. The quality and abundance of ground water depends upon depth of water table, ecological factors and presence of dissolved solids. In India and certain parts of the world, water crisis is becoming a regular phenomenon, perhaps due to improper scientific management of water resources leading to Author's Address

${ }^{1}$ Department of Zoology and Environmental Science, Gurukul Kangri Vishwavidyalaya, Haridwar

${ }^{2}$ Asst. Registrar, Gurukul Kangri Vishwavidyalaya, Haridwar E-mail.: khushi2013ram@gmail.com

Copyright by ASEA

All rights of reproduction in any form reserved continued environmental degradation. In the last few decades, there has been a tremendous increase in the demand for freshwater resource due to rapid population growth and industrialization (Rama Krishna et al., 2009). The quality and depletion of ground water in most countries including India is directly affected by population, large scale disposal of urban and industrial wastes and use of chemical fertilizers and pesticides in agricultural fields (CGWB and CPCB, 2000; CGWB, 1999, 2000, 2001; Sharma and Jain, 2006). The ground water aquifers are the major source of the drinking water and important sources of surface water. Due to increased pollution, water becoming unsafe for consumption. The urban aquatic ecosystems are strongly influenced by long term discharge of untreated domestic and industrial wastewaters, storm water runoff, accidental spills and direct solid waste dumping. All these released pollutants have great ecological impacts on water quality of urban wetlands.

\section{Material and Methods}

Study area: The study was conducted in the Laksar Block of Haridwar, Uttarakhand. Laksar is a town and a panchayat in Haridwar district of Uttarakhand. It is an important sugar manufacturing 
destination in the state. Laksar has an average elevation of 227 metres (745 feet). It is situated between the towns of Khanpur and Sultanpur, and close to the towns of Pathri, Jhabrera and Roorkee in Haridwar district. The water samples were collected from all sites in the jerry cans of 2 liters capacity. Spot sampling was used for samples collection. Samples were analyzed for the following physic-chemical parameters. Ground water quality monitoring was done at each locations and ground water samples were taken from private hand pump (30-40 feet), government hand pump (50-60 feet), as per CPCB guideline for Water Quality Monitoring. Two samples were taken from each of the locations once a month and analysed for various physico-chemical parameters with the help of APHA, 2012; Trivedy and Goel, 1986 and Khanna and Bhutiani, 2008.
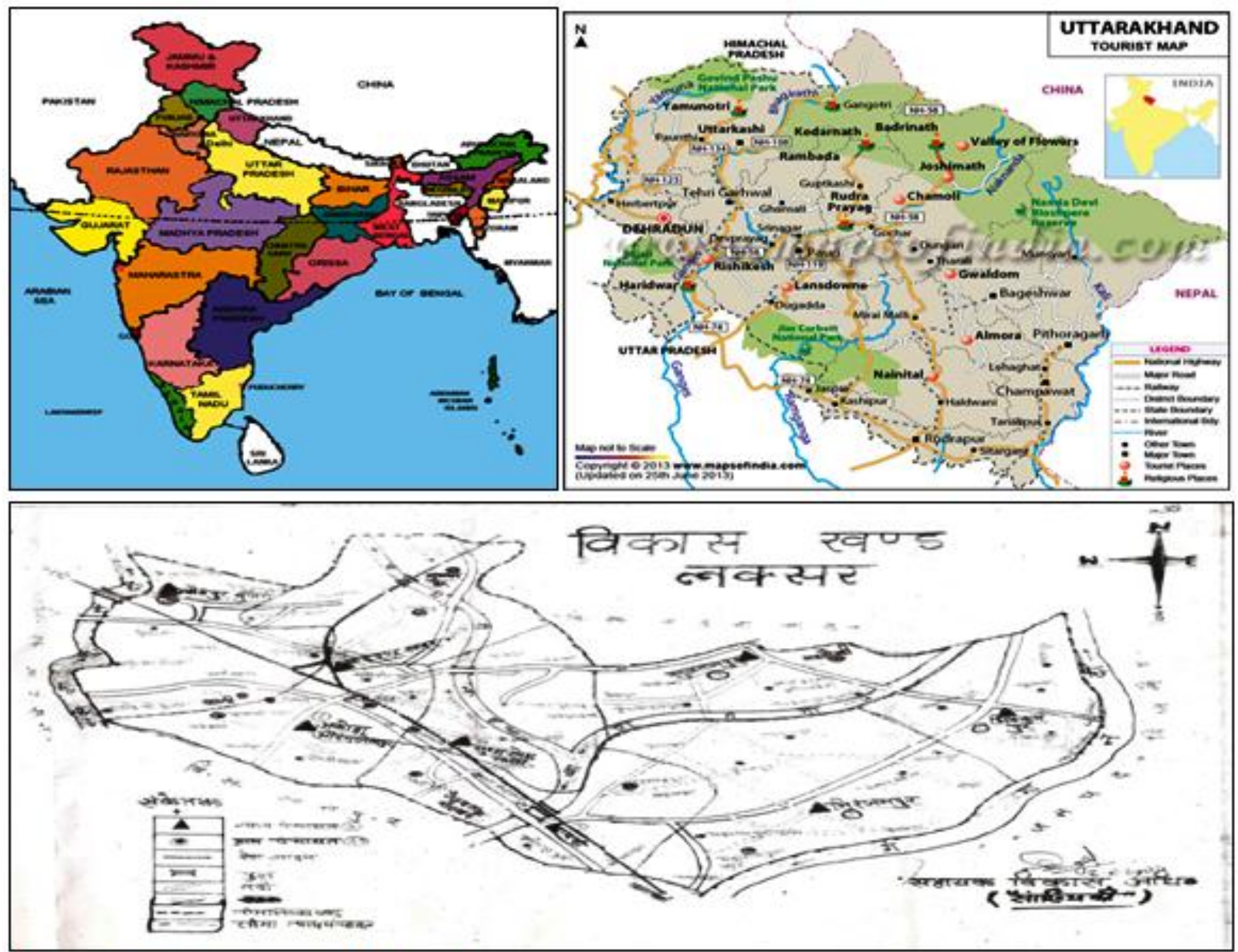

Figure.1 Location map of the study area

Table 1: Different sites selected for sampling of ground water

\begin{tabular}{|l|l|l|}
\hline Sites & Latitude & Longitudes \\
\hline SS-1 Laksar & $29^{\circ} 45^{\prime} 13.8348^{\prime \prime} \mathrm{N}$ & $78^{\circ} 11^{\prime} 17.3352^{\prime \prime} \mathrm{E}$ \\
\hline SS-2 DabkiKalan & $29^{\circ} 44^{\prime} 29.256^{\prime \prime} \mathrm{N}$ & $78^{\circ} 0^{\prime} 39.5136 " \mathrm{E}$ \\
\hline SS-3 Akaudha & $29^{\circ} 44^{\prime} 42.3924^{\prime \prime} \mathrm{N}$ & $78^{\circ} 3{ }^{\prime} 3.3408 " \mathrm{E}$ \\
\hline SS-4 Khandia & $29^{\circ} 43^{\prime} 51.6936^{\prime \prime} \mathrm{N}$ & $78^{\circ} 2^{\prime} 33.846 " \mathrm{E}$ \\
\hline SS-5 Raisi & $29^{\circ} 41^{\prime} 58.0128^{\prime \prime} \mathrm{N}$ & $78^{\circ} 4^{\prime} 49.17^{\prime \prime} \mathrm{E}$ \\
\hline SS-6 Niranjanpur & $30^{\circ} 17^{\prime} 50.6436^{\prime \prime} \mathrm{N}$ & $78^{\circ} 0^{\prime} 36.4068^{\prime \prime} \mathrm{E}$ \\
\hline
\end{tabular}




\section{Results and Discussion}

Monthly average value of different parameters, Temperature, TS, TDS, EC, Turbidity, pH, DO, Total Hardness, Calcium Hardness, Chloride, Iron, Sulphate, Acidity presented was Given in table 1and2 respectively.

Temperature: During the present study, the minimum temperature $\left(24.4 \quad \pm 3.26{ }^{0} \mathrm{C}\right)$ was recorded from the samples of SS-3, while the maximum $\left(25.6 \pm 30{ }^{0} \mathrm{C}\right)$ from PHP of SS-1site. Similarly, the samples collected from GHP show the minimum temperature $\left(24.4 \pm 3.2{ }^{\circ} \mathrm{C}\right)$ at SS-3, while the maximum $\left(25.7 \pm 3.33^{\circ} \mathrm{C}\right)$ at $\mathrm{SS}-1$ and monthly average value of temperature was 25.0 $\pm 0.4^{0} \mathrm{C}$. A more or less similar trend was observed by Tyagi et al., 2015 .

TS: During the present study in samples of PHP, the minimum total solid (TS) value was 705.3 \pm 71.5 $\mathrm{mg} / \mathrm{l}$ recorded from at SS-6 while the maximum $(789.0 \pm 30.2 \mathrm{mg} / \mathrm{l})$ from at SS-1 site. The monthly average value of total solid (TS) was $763.8 \pm 30.3$ $\mathrm{mg} / \mathrm{l}$. Similarly the minimum total solid (TS) value was found $696.1 \mathrm{mg} / 1 \pm 71.0$ at GHP of at SS-6 while the maximum value $(783.2 \pm 35.7 \mathrm{mg} / \mathrm{l})$ was recorded from at SS-1. The monthly average value of total solid (TS) was $760.2 \pm 33.7 \mathrm{mg} / \mathrm{l}$. Similar results were reported by Bhadja and Vaghela, 2013. TDS: During the present study In the PHP, the minimum total dissolve solid (TDS) value was found $539.8 \pm 45.55 \mathrm{mg} / \mathrm{l}$ at $\mathrm{SS}-1$ while the maximum was found $580.4 \pm 54.3 \mathrm{mg} / \mathrm{l}$ at $\mathrm{SS}-5$ and monthly average value was observed $534.9 \pm 38.6$ $\mathrm{mg} / \mathrm{l}$ while in GHP, the minimum total dissolve solid (TDS) value was found. $454 \pm 82.5 \mathrm{mg} / \mathrm{l}$ at SS6 while in the maximum was found $573.8 \pm 28.7$ $\mathrm{mg} / \mathrm{l}$ at SS-2 and monthly average value was observed $524.5 \pm 40.2 \mathrm{mg} / \mathrm{l}$ Similar results were reported by kumar et al., 2017.

EC: During the present study In the PHP, the minimum electronic conductivity value was found $1343.5 \pm 8.7$ (micro mho $\mathrm{cm}-1$ ) at SS-6 while the maximum was found $1467.7 \pm 3.6$ (micro mho $\mathrm{cm}-1$ ) at SS-3 and monthly average value of electronic conductivity was observed 1400.01 (micro mho $\mathrm{cm}-1) \pm 42.6$ While in GHP the minimum electronic conductivity value was found $1345.2 \pm 0.6$ (micro mho $\mathrm{cm}-1$ ) at SS-6 while the maximum was found $1469.6 \pm 17.2$ (micro $m h o ~ c m$ 1) at SS-3 and monthly average value of was

observed $1398.81 \pm 44.0$ (micro mho cm-1) similar results were observed by Zawar et al., 2015.

Turbidity: Turbidity is an important indicator, which determines the amount of suspended sediment in water. These particles can block light to aquatic plants, smother aquatic organisms, and carry contaminants and pathogens, such as lead, mercury, and bacteria. During this study, the minimum turbidity value $(1.11 \pm 0.6 .7 \mathrm{NTU})$ was recorded in samples from the PHP of at SS-6, while the maximum $(5.1 \pm 2 \mathrm{NTU})$ at SS-1 site. The monthly average value was $3.71 \pm 1.4$ NTU. The minimum turbidity $(0.33 \pm 0.7$ NTU $)$ was recorded from the samples of GHP at SS-6 site, while the maximum $(4.33 \pm 2.0 \mathrm{NTU})$ at SS-4. The monthly average value observed 3.31 NTU \pm 1.63 . Similar trend was found by Mandloi, 2014 and Bhutiani et al., 2015.

pH: During the present study In the PHP, the minimum $\mathrm{pH}$ value was found $6.9 \pm 0.5$ at SS-6 while the maximum was found $7.7 \pm 0.6$ at SS- 1 and private hand pump monthly average value of $\mathrm{pH}$ was observed $7.11 \pm 0.3$ while in GHP, the minimum $\mathrm{pH}$ value was found. $6.8 \pm 0.6$ at SS-6 while the maximum was found $7.5 \pm 0.1$ at SS-5 and GHP monthly average value of $\mathrm{pH}$ was observed $7.11 \pm 0.3$ Similar trend was found by Saxena and Saxena 2015.

DO: During the present study In the PHP, the minimum DO value was found $6.1 \pm 0.5 \mathrm{mg} / \mathrm{l}$ at SS-2 while the maximum was found $6.5 \pm 0.5 \mathrm{mg} / \mathrm{l}$ at SS-6 monthly average value of DO was observed $6.2 \pm 0.1 \mathrm{mg} / \mathrm{l}$ while in GHP, the minimum DO value was found. $6.1 \mathrm{mg} / \mathrm{l} \pm 0.5 \mathrm{mg} / \mathrm{l}$ at SS-3 while the maximum was found $6.6 \pm 0.5$ $\mathrm{mg} / \mathrm{l}$ at SS-6 and monthly average value of DO was observed $6.2 \pm 0.2 \mathrm{mg} / \mathrm{l} \mathrm{Similar}$ trend was found by jai et al., 2014.

Total Hardness: During the present study In the PHP, the minimum total hardness (TH) value was found $330.5 \pm 42.4 \mathrm{mg} / \mathrm{l}$ at SS-6 while the maximum was found $396.8 \pm 65.9 \mathrm{mg} / \mathrm{l}$ at SS-5 and monthly average value of total hardness $(\mathrm{TH})$ was observed $\quad 374.2 \pm 22.6 \mathrm{mg} / \mathrm{l}$ while in GHP the minimum at total hardness(TH) value was found. $325.7 \pm 46.2 \mathrm{mg} / \mathrm{l}$ at SS-6 while the maximum was found $377.0 \pm 36.2 \mathrm{mg} / \mathrm{l}$ at SS-1 and monthly average value was observed $370.6 \pm 23.4 \mathrm{mg} / \mathrm{l}$ similar result reported by Ramakrishnaiah et al., 2009. 
Table 2. Physical characteristics of ground water of different sites of Laksar Block.

\begin{tabular}{|c|c|c|c|c|c|c|c|c|c|c|c|c|c|c|}
\hline \multirow{2}{*}{$\begin{array}{c}\text { Sites/ } \\
\text { Parameters }\end{array}$} & \multicolumn{2}{|c|}{ Laksar } & \multicolumn{2}{|c|}{ Dabki kalan } & \multicolumn{2}{|c|}{ Akaudha } & \multicolumn{2}{|c|}{ Khandja } & \multicolumn{2}{|c|}{ Raisi } & \multicolumn{2}{|c|}{ Niranjanpur } & \multicolumn{2}{|c|}{ Average } \\
\hline & PHP & $\mathbf{G}$ & $\mathbf{P}$ & $\mathbf{G}$ & $\mathbf{P}$ & $\mathbf{G}$ & $\mathbf{P}$ & $\mathbf{G}$ & $\mathbf{P}$ & $\mathbf{G}$ & $\mathbf{P}$ & $\mathbf{G}$ & $\mathbf{P}$ & $\mathbf{G}$ \\
\hline Temperature & $\begin{array}{l}25.6 \\
\pm 3.1\end{array}$ & $\begin{array}{l}25.7 \\
\pm 3.3\end{array}$ & $\begin{array}{l}24.8 \\
\pm 3.3\end{array}$ & $\begin{array}{l}24.7 \\
\pm 3.3\end{array}$ & $\begin{array}{l}24.8 \\
\pm 3.2\end{array}$ & $\begin{array}{c}24.4 \pm \\
3.2\end{array}$ & $\begin{array}{l}25.1 \\
\pm 3.4\end{array}$ & $\begin{array}{l}24.9 \\
\pm 3.4\end{array}$ & $\begin{array}{l}25.1 \\
\pm 3.5\end{array}$ & $\begin{array}{l}25.2 \\
\pm 3.4\end{array}$ & $\begin{array}{l}24.9 \\
\pm 3.4\end{array}$ & $\begin{array}{l}24.9 \\
\pm 3.4\end{array}$ & $\begin{array}{l}25.1 \\
\pm 0.3\end{array}$ & $\begin{array}{l}25.0 \\
\pm 0.4\end{array}$ \\
\hline Total Solids (TS) & $\begin{array}{r}789.0 \\
\pm 30.2 \\
\end{array}$ & $\begin{array}{r}783.2 \\
\pm 35.7 \\
\end{array}$ & $\begin{array}{l}770.4 \\
\pm 13.6 \\
\end{array}$ & $\begin{array}{r}771.9 \\
\pm 20.1 \\
\end{array}$ & $\begin{array}{l}777.0 \\
\pm 15.4 \\
\end{array}$ & $\begin{array}{r}774.6 \\
\pm 20.8 \\
\end{array}$ & $\begin{array}{r}780.9 \\
\pm 10.7 \\
\end{array}$ & $\begin{array}{l}778.6 \\
\pm 18.8 \\
\end{array}$ & $\begin{array}{l}760.1 \\
\pm 40.7\end{array}$ & $\begin{array}{l}751.4 \\
\pm 33.5\end{array}$ & $\begin{array}{l}705.3 \\
\pm 71.5\end{array}$ & $\begin{array}{l}696.1 \\
\pm 71.0 \\
\end{array}$ & $\begin{array}{l}763.8 \\
\pm 30.3\end{array}$ & $\begin{array}{l}760.2 \\
\pm 33.7\end{array}$ \\
\hline Total Dissolved Solids (TDS) & $\begin{array}{l}539.8 \\
\pm 45.5 \\
\end{array}$ & $\begin{array}{l}528.2 \\
\pm 58.5 \\
\end{array}$ & $\begin{array}{l}535.8 \\
\pm 32.9 \\
\end{array}$ & $\begin{array}{l}573.8 \\
\pm 28.7 \\
\end{array}$ & $\begin{array}{l}541.6 \\
\pm 38.6 \\
\end{array}$ & $\begin{array}{l}536.4 \\
\pm 40.8 \\
\end{array}$ & $\begin{array}{l}548.6 \\
\pm 30.9 \\
\end{array}$ & $\begin{array}{l}543.3 \\
\pm 30.4 \\
\end{array}$ & $\begin{array}{l}580.4 \\
\pm 54.3 \\
\end{array}$ & $\begin{array}{l}511.2 \\
\pm 62.2 \\
\end{array}$ & $\begin{array}{l}463.3 \\
\pm 83.8 \\
\end{array}$ & $\begin{array}{c}454 \\
\pm 82.5 \\
\end{array}$ & $\begin{array}{l}534.9 \\
\pm 38.6 \\
\end{array}$ & $\begin{array}{l}524.5 \\
\pm 40.2 \\
\end{array}$ \\
\hline Electrical Conductiuvity (EC) & $\begin{array}{c}1379.0 \\
\pm 23.0 \\
\end{array}$ & $\begin{array}{c}1366.0 \\
\pm 20.8 \\
\end{array}$ & $\begin{array}{c}1423.8 \\
\pm 4.2\end{array}$ & $\begin{array}{c}1422.0 \\
\pm 8.0\end{array}$ & $\begin{array}{c}1467.7 \\
\pm 3.6\end{array}$ & $\begin{array}{c}1469.0 \\
\pm 17.2 \\
\end{array}$ & $\begin{array}{c}1382.0 \\
\pm 10.2 \\
\end{array}$ & $\begin{array}{r}1386 \\
\pm 13.7 \\
\end{array}$ & $\begin{array}{c}1403.0 \\
\pm 7.8\end{array}$ & $\begin{array}{l}1402.0 \\
\pm 14.1\end{array}$ & $\begin{array}{c}1343.0 \\
\pm 8.7\end{array}$ & $\begin{array}{c}1345.0 \\
\pm 0.6\end{array}$ & $\begin{array}{l}1400.0 \\
. \pm 42.6\end{array}$ & $\begin{array}{l}1398.0 \\
\pm 44.0\end{array}$ \\
\hline Turbidity & $\begin{array}{c}51.00 \\
\pm 2 .\end{array}$ & $\begin{array}{l}5.00 \\
\pm 2.1\end{array}$ & $\begin{array}{l}4.44 \\
\pm 1.9\end{array}$ & $\begin{array}{l}3.55 \\
\pm 2.4\end{array}$ & $\begin{array}{l}3.66 \\
\pm 2.2\end{array}$ & $\begin{array}{l}3.66 \\
\pm 2.2\end{array}$ & $\begin{array}{l}4.33 \\
\pm 2.0\end{array}$ & $\begin{array}{l}4.33 \\
\pm 2.0\end{array}$ & $\begin{array}{l}3.55 \\
\pm 1.5\end{array}$ & $\begin{array}{l}3.15 \\
\pm 1.7\end{array}$ & $\begin{array}{l}1.11 \\
\pm 0.6\end{array}$ & $\begin{array}{l}0.33 \\
\pm 0.7\end{array}$ & $\begin{array}{l}3.71 \\
\pm 1.4\end{array}$ & $\begin{array}{l}3.31 \\
\pm 1.6\end{array}$ \\
\hline
\end{tabular}

Table 3. Chemical characteristics of ground water of different sites of Laksar Block.

\begin{tabular}{|c|c|c|c|c|c|c|c|c|c|c|c|c|c|c|}
\hline \multirow[t]{2}{*}{ Sites/Parameters } & \multicolumn{2}{|c|}{ Laksar } & \multicolumn{2}{|c|}{ Dabki kalan } & \multicolumn{2}{|c|}{ Akaudha } & \multirow{2}{*}{$\begin{array}{c}\text { Khandja } \\
P\end{array}$} & \multicolumn{2}{|c|}{ Raisi } & \multicolumn{2}{|c|}{ Niranjanpur } & \multicolumn{3}{|c|}{ Average } \\
\hline & $\mathbf{P}$ & $\mathbf{G}$ & $\mathbf{P}$ & $\mathbf{G}$ & $\mathbf{P}$ & $\mathbf{G}$ & & $\mathbf{G}$ & $\mathbf{P}$ & $\mathbf{G}$ & $\mathbf{P}$ & $\mathbf{G}$ & $\mathbf{P}$ & $\mathbf{G}$ \\
\hline$\overline{\mathrm{pH}}$ & $\begin{array}{c}7.7 \\
\pm 0.6 \\
\end{array}$ & $\begin{array}{c}7.3 \\
\pm 0.61 \\
\end{array}$ & $\begin{array}{c}6.9 \\
\pm 0.7 \\
\end{array}$ & $\begin{array}{c}6.9 \\
\pm 0.7 \\
\end{array}$ & $\begin{array}{c}7.0 \\
\pm 0.6 \\
\end{array}$ & $\begin{array}{c}7.0 \\
\pm 0.7 \\
\end{array}$ & $\begin{array}{c}7.0 \\
\pm 0.8 \\
\end{array}$ & $\begin{array}{c}7.2 \\
\pm 0.7 \\
\end{array}$ & $\begin{array}{c}7.3 \\
\pm 0.2 \\
\end{array}$ & $\begin{array}{c}7.5 \\
\pm 0.1 \\
\end{array}$ & $\begin{array}{c}6.9 \\
\pm 0.5 \\
\end{array}$ & $\begin{array}{c}6.8 \\
\pm 0.6 \\
\end{array}$ & \begin{tabular}{|c|}
$7.11 \pm$ \\
0.3 \\
\end{tabular} & \begin{tabular}{|l|}
7.11 \\
\pm 0.3 \\
\end{tabular} \\
\hline Dissolved Oxygen (DO) & $\begin{array}{c}6.2 \\
\pm 0.5 \\
\end{array}$ & $\begin{array}{c}6.2 \\
\pm 0.5\end{array}$ & $\begin{array}{c}6.1 \\
\pm 0.5 \\
\end{array}$ & $\begin{array}{c}6.2 \\
\pm 0.5\end{array}$ & $\begin{array}{c}6.1 \\
\pm 0.4\end{array}$ & $\begin{array}{c}6.1 \\
\pm 0.5\end{array}$ & $\begin{array}{c}6.2 \\
\pm 0.5\end{array}$ & $\begin{array}{c}6.1 \\
\pm 0.5\end{array}$ & $\begin{array}{c}6.2 \\
\pm 0.5\end{array}$ & $\begin{array}{c}6.2 \\
\pm 0.5 \\
\end{array}$ & $\begin{array}{c}6.5 \\
\pm 0.5\end{array}$ & $\begin{array}{c}6.6 \\
\pm 0.5\end{array}$ & $\begin{array}{c}6.2 \\
\pm 0.1\end{array}$ & $\begin{array}{c}6.2 \\
\pm 0.2 \\
\end{array}$ \\
\hline Total Hardness (TH) & $\begin{array}{l}381.8 \\
\pm 32.8 \\
\end{array}$ & $\begin{array}{l}377.0 \\
\pm 36.2 \\
\end{array}$ & $\begin{array}{l}377.7 \\
\pm 29.0 \\
\end{array}$ & $\begin{array}{l}373.2 \\
\pm 32.6 \\
\end{array}$ & $\begin{array}{l}377.6 \\
\pm 26.4 \\
\end{array}$ & $\begin{array}{l}377.1 \\
\pm 31.8 \\
\end{array}$ & $\begin{array}{l}380.7 \\
\pm 27.5 \\
\end{array}$ & $\begin{array}{l}374.4 \\
\pm 23.7 \\
\end{array}$ & $\begin{array}{l}396.8 \\
\pm 65.9 \\
\end{array}$ & $\begin{array}{l}395.2 \\
\pm 61.5 \\
\end{array}$ & $\begin{array}{l}330.5 \\
\pm 42.4 \\
\end{array}$ & $\begin{array}{l}325.7 \\
\pm 46.2 \\
\end{array}$ & \begin{tabular}{|l|}
374.2 \\
\pm 22.6 \\
\end{tabular} & $\begin{array}{l}370.6 \\
\pm 23.4 \\
\end{array}$ \\
\hline Calcium Hardness (CaH) & $\begin{array}{l}331.3 \\
\pm 32.5\end{array}$ & $\begin{array}{l}325.1 \\
\pm 34.5\end{array}$ & $\begin{array}{l}329.1 \\
\pm 31.5\end{array}$ & $\begin{array}{l}324.0 \\
\pm 35.9\end{array}$ & $\begin{array}{r}327.3 \\
\pm 30.8 \\
\end{array}$ & $\begin{array}{l}320.5 \\
\pm 37.7 \\
\end{array}$ & $\begin{array}{l}330.0 \\
\pm 31.0 \\
\end{array}$ & $\begin{array}{l}323.7 \\
\pm 33.3\end{array}$ & $\begin{array}{l}326.4 \\
\pm 57.9\end{array}$ & $\begin{array}{l}315.5 \\
\pm 49.4\end{array}$ & $\begin{array}{l}290.7 \\
\pm 57.0 \\
\end{array}$ & $\begin{array}{l}288.7 \\
\pm 55.5 \\
\end{array}$ & \begin{tabular}{|l|}
322.5 \\
\pm 15.7 \\
\end{tabular} & \begin{tabular}{|l|}
316.3 \\
\pm 13.9 \\
\end{tabular} \\
\hline Chloride (Cl) & $\begin{array}{c}137.3 \\
\pm 8.7 \\
\end{array}$ & $\begin{array}{l}132.2 \\
\pm 10.2 \\
\end{array}$ & $\begin{array}{c}125.0 \\
\pm 5.6 \\
\end{array}$ & $\begin{array}{c}123.7 \\
\pm 7.5 \\
\end{array}$ & $\begin{array}{c}119.1 \\
\pm 7.2 \\
\end{array}$ & $\begin{array}{l}116.4 \\
\pm 11.3 \\
\end{array}$ & $\begin{array}{c}114.0 \\
\pm 5.8 \\
\end{array}$ & $\begin{array}{c}113.5 \\
\pm 7.2 \\
\end{array}$ & $\begin{array}{l}96.6 \\
\pm 8.6 \\
\end{array}$ & $\begin{array}{c}95.0 \\
\pm 11.9 \\
\end{array}$ & $\begin{array}{r}90.6 \\
\pm 9.9 \\
\end{array}$ & $\begin{array}{c}90.2 \\
\pm 14.1 \\
\end{array}$ & \begin{tabular}{|l|}
113.8 \\
\pm 17.5 \\
\end{tabular} & \begin{tabular}{|l|}
111.8 \\
\pm 16.3 \\
\end{tabular} \\
\hline Acidity & $\begin{array}{l}89.7 \\
\pm 6.2 \\
\end{array}$ & $\begin{array}{l}89.4 \\
\pm 6.2 \\
\end{array}$ & $\begin{array}{l}82.0 \\
\pm 5.4 \\
\end{array}$ & $\begin{array}{l}82.7 \\
\pm 4.4 \\
\end{array}$ & $\begin{array}{l}86.4 \\
\pm 8.1 \\
\end{array}$ & $\begin{array}{l}86.0 \\
\pm 6.1 \\
\end{array}$ & $\begin{array}{r}78.4 \\
\pm 4.4 \\
\end{array}$ & $\begin{array}{r}77.7 \\
\pm 4.0 \\
\end{array}$ & $\begin{array}{r}76.4 \\
\pm 8.3 \\
\end{array}$ & $\begin{array}{r}75.4 \\
\pm 6.8 \\
\end{array}$ & $\begin{array}{r}63.3 \\
\pm 5.2 \\
\end{array}$ & $\begin{array}{r}61.1 \\
\pm 3.8 \\
\end{array}$ & \begin{tabular}{|l|}
79.4 \\
\pm 9.3 \\
\end{tabular} & \begin{tabular}{|c|}
78.7 \\
\pm 10.1 \\
\end{tabular} \\
\hline Iron $(\mathrm{Fe})$ & $\begin{array}{c}0.3 \\
\pm 0.0 \\
\end{array}$ & $\begin{array}{c}0.3 \\
\pm 0.0 \\
\end{array}$ & $\begin{array}{c}0.3 \\
\pm 0.0 \\
\end{array}$ & $\begin{array}{c}0.3 \\
\pm 0.0 \\
\end{array}$ & $\begin{array}{c}0.4 \\
\pm 0.0 \\
\end{array}$ & $\begin{array}{c}0.3 \\
\pm 0.0 \\
\end{array}$ & $\begin{array}{c}0.3 \\
\pm 0.0 \\
\end{array}$ & $\begin{array}{c}0.3 \\
\pm 0.0 \\
\end{array}$ & $\begin{array}{c}0.2 \\
\pm 0.0 \\
\end{array}$ & $\begin{array}{c}0.2 \\
\pm 0.0 \\
\end{array}$ & $\begin{array}{c}0.2 \\
\pm 0.0 \\
\end{array}$ & $\begin{array}{c}0.2 \\
\pm 0.1 \\
\end{array}$ & $\begin{array}{c}0.3 \\
\pm 0.1 \\
\end{array}$ & \begin{tabular}{c|}
0.3 \\
\pm 0.1 \\
\end{tabular} \\
\hline Sulphate $\left(\mathrm{SO}_{4}\right)$ & $\begin{array}{r}40.2 \\
\pm 3.1\end{array}$ & $\begin{array}{l}39.0 \\
\pm 4.9\end{array}$ & $\begin{array}{r}40.2 \\
\pm 1.5\end{array}$ & $\begin{array}{l}38.0 \\
\pm 2.3\end{array}$ & $\begin{array}{r}42.4 \\
\pm 1.8\end{array}$ & $\begin{array}{l}41.0 \\
\pm 5.4\end{array}$ & $\begin{array}{l}45.1 \\
\pm 0.7\end{array}$ & $\begin{array}{r}42.2 \\
\pm 1.4\end{array}$ & $\begin{array}{c}42.1 \\
\pm 42.1\end{array}$ & $\begin{array}{c}41.3 \\
\pm 41.3\end{array}$ & $\begin{array}{l}32.3 \\
\pm 5.6\end{array}$ & $\begin{array}{l}30.2 \\
\pm 6.4\end{array}$ & $\begin{array}{r}40.4 \\
\pm 4.4\end{array}$ & $\begin{array}{r}38.8 \\
\pm 4.4\end{array}$ \\
\hline
\end{tabular}

PHP- Private hand pump and GHP- Government hand pump 
Calcium Hardness: During the present study In the PHP, the minimum calcium hardness $(\mathrm{CaH})$ value was found $290.7 \pm 57.0 \mathrm{mg} / \mathrm{l}$ at SS- 6 while the maximum was found $331.3 \pm 32.5 \mathrm{mg} / \mathrm{l}$ at SS-1 and monthly average value of calcium hardness $(\mathrm{CaH})$ was observed $322.5 \pm 15.7 \mathrm{mg} / \mathrm{l}$ while in GHP the minimum at calcium hardness $(\mathrm{CaH})$ value was found. $288.7 \pm 55.5 \mathrm{mg} / \mathrm{l}$ at $\mathrm{SS}-6$ while the maximum was found $325.1 \pm 34.5 \mathrm{mg} / \mathrm{l}$ at SS-1 and monthly average value was observed 316.3 $\pm 13.9 . \mathrm{mg} / \mathrm{l}$. Similar results were reported by Shankar, 2007.

Chloride: During the present study In the PHP, the minimum Chloride value was found $90.6 \pm 9.9 \mathrm{mg} / \mathrm{l}$ at SS-6 while the maximum was found $137.3 \pm 8.7 \mathrm{mg} / \mathrm{l}$ at SS- 1 and monthly average value of Chloride was observed $113.8 \pm 17.5 \mathrm{mg} / \mathrm{l}$ while in GHP, the minimum Chloride value was found. 90.2 $\pm 14.1 \mathrm{mg} / \mathrm{l}$ at SS-6 while the maximum was found $132.2 \pm 10.2 \mathrm{mg} / \mathrm{l}$ at SS-1 and monthly average value was observed $111.8 \pm 16.3 \mathrm{mg} / \mathrm{l}$. Similar trend was found by Saxena and Saxena, 2015.

Acidity: During the present study In the PHP, the minimum Acidity value was found $63.3 \pm 5.2 \mathrm{mg} / \mathrm{l}$ at SS-6 while the maximum was found $89.7 \pm 6.2$ $\mathrm{mg} / \mathrm{l}$ at SS-1 and private hand pump monthly average value of Acidity was observed $79.4 \pm 9.3$ $\mathrm{mg} / \mathrm{l}$ while in GHP , the minimum at Acidity value was found. $61.1 \pm 3.8 \mathrm{mg} / \mathrm{l}$ at SS-6 while the maximum value was found $89.4 \pm 6.2 \mathrm{mg} / \mathrm{l}$ at SS-1 and monthly average value of was observed 78.7 $\pm 10.1 \mathrm{mg} / \mathrm{l}$

Iron: During the present study In the PHP, the minimum Iron value was found $0.21 \pm 0.0 \mathrm{mg} / \mathrm{l}$ at SS-5 while the maximum was found $0.4 \pm 0.0 \mathrm{mg} / \mathrm{l}$ at SS-3 and monthly average value of Iron was observed $0.3 \pm 0.1 \mathrm{mg} / \mathrm{l}$ while in GHP, the minimum at Iron value was found. $0.2 \pm 0.0 \mathrm{mg} / \mathrm{l}$ at SS-5 while the maximum was found $0.3 \pm 0.0 \mathrm{mg} / \mathrm{l}$ at SS-1 and monthly average value of Iron was observed $0.3 \pm 0.1 \mathrm{mg} / \mathrm{l}$ Similar result were observed by Zawar et al., 2015.

Sulphate: During the present study In the PHP, the minimum Sulphate value was found $32.3 \pm 5.6 \mathrm{mg} / \mathrm{l}$ at SS-6 while the maximum was found. $45.1 \pm 0.7$ $\mathrm{mg} / \mathrm{l}$ at SS-4 and private hand pump monthly average value of Sulphate was observed $40.4 \pm 4.4$ $\mathrm{mg} / \mathrm{l}$ while in GHP, the minimum at Sulphate value was found $30.2 \pm 6.4 \mathrm{mg} / \mathrm{l}$ at SS-6 while the maximum was found. $42.2 \pm 1.4 \mathrm{mg} / \mathrm{l}$ at SS-4 and GHP monthly average value of Sulphate was observed $38.8 \pm 4.4 \mathrm{mg} / \mathrm{l}$ Similar trend was found by Mandloi 2014.

Table 4. Showing average values of different parameters and standard limits of WHO and BIS

\begin{tabular}{|l|c|c|c|c|}
\hline \multicolumn{1}{|c|}{ Parameter } & PHP & GHP & WHO & BIS \\
\hline Temperature & 25.1 & 25.0 & - & - \\
\hline Total Solids (TS) & 763.8 & 760.2 & - & - \\
\hline Total Dissolved Solids (TDS) & 534.9 & 524.5 & $500 \mathrm{mg} / \mathrm{l}$ & $500 \mathrm{mg} / \mathrm{l}$ \\
\hline Electrical Conductiuvity (EC) & 1400 & 1398 & & \\
\hline Turbidity & 3.71 & 3.31 & $5.0 \mathrm{NTU}$ & $5.0 \mathrm{NTU}$ \\
\hline pH & 7.11 & 7.11 & $7.0-8.5$ & $6.5-8.5$ \\
\hline Dissolved Oxygen (DO) & 6.2 & 6.2 & 14 & - \\
\hline Total Hardness (TH) & 374.2 & 370.6 & 200 & - \\
\hline Calcium Hardness (CaH) & 322.5 & 316.3 & - & 75 \\
\hline Chloride (CI) & 113.8 & 111.8 & 250 & 250 \\
\hline Acidity & 79.4 & 78.7 & - & - \\
\hline Iron (Fe) & 0.3 & 0.3 & $0.3 \mathrm{mg} / \mathrm{l}$ & $0.3 \mathrm{mg} / \mathrm{l}$ \\
\hline Sulphate $\left(\mathbf{S O}_{\mathbf{4}}\right)$ & 40.4 & 38.8 & - & 200 \\
\hline
\end{tabular}

\section{Conclusion}

During the present study total dissolved solids (TDS) was found slightly higher than the limits of WHO and BIS while calcium hardness $(\mathrm{CaH})$ was found approximately four to five times higher than the limits of WHO and BIS. The average values of iron was presently found equal to the limits of WHO and BIS but at some sites the values of iron was found above the limits of WHO and BIS. Rest of the parameters analyzed during the study such as Temperature, TS, EC, PH, DO, Total Hardness (TH), Chloride Acidity and Sulphate was found 
under the limit of WHO and BIS. On the basis of clinical survey it was found that peoples were suffering from different water related disease such as typhoid, Dysentery and kidneys stones. Some allergic problems were also reported during the study period. On the basis of present study we can conclude that area under the present study was in normal condition presently with reference to ground water pollution but if the trend of industrialization will continue, it will result in bad condition of ground water.

\section{References}

APHA., 2012. "Standard Methods for the examination of Water and Wastewater", Lenore S C, Greenberg A E, Eaton A D,(Eds.), 20th Edition, American Public Health Association, NW, Washington, DC.

Arya,V.S, SainiAakash, Hardev Singh and Hooda, R.S., 2011. Watershed Based Ground Water Quality Studies UsingGeo ]Informatics in Mahendergarh District,Haryana,Bhujal25:(2\&3) and 26:(1-4) (Combined)

Bhadja Poonam and Vaghela Ashok kumar, 2013. Assessment of physico-chemical parameters and water quality index of reservoir water, international journal of plant, animal and environmental sciences, 3(3):89-95.

Bhutiani, R., Kulkarni, D. B., Khanna D. R., and Gautam A., 2016. Water Quality, Pollution Source Apportionment and Health Risk Assessment of Heavy Metals in Groundwater of an Industrial Area in North India. Expo Health, 8: 3-18.

Bhutiani R., Khanna D.R., Tyagi Varun, Ahamad Faheem, 2015.Removal of turbidity in dairy waste water through aquatic macrophytes, International Journal of Research GRANTHAALAYAH. 9(3):1-3.

CGWB 1999. Hydrogeological Framework for Urban Development of Bhopal City, Madhya Pradesh, Central Ground Water Board, North Central Region, Bhopal, India.

CGWB 2000. Ground Water Quality in Urban Environment of India, Central Ground Water Board, Faridabad, India

CGWB 2001.Hydrogeological Framework and Ground Water development Prospects in Jabalpur City, M.P., Central Ground Water Board, North Central Region, Bhopal, India.

CGWB and CPCB 2000. Status of Ground Water Quality and Pollution Aspects in NCT-Delhi, India.

Das, S., Metha, B. C., Das, P. K., Srivastava, S. K. and Samanth, S. K. 1998.Source of high fluoride in ground water of Anugul, Dhenkenal District, Orrissa.Pollution Research, 17(4):385-392.
Jai M Paul, Arya V.S, Jesteena George, Reji K.. J. and Sumitha K. S., 2014. Assessment of Ground Water Quality in Nellikkuzhy Panchayat of Kerala State, India, International Journal of Engineering Science Invention, 3 (4) :21-28.

Khanna,D.R., and Bhutiani, R., 2008. Laboratory manual of water and Waste water Analysis. Daya Publishing House New Delhi -110002.

Kumar Sunil, Kumar Manish, Kumar Anuradha, Kumar Amit and Singh A.K. 2017. Effect of Sugar Mill Effluents on Ground Water Quality Int.J.Curr.Microbiol.App.Sci, 6(12): 169-176 .

Mandloi Abhishek. 2014. Impact of industries on ground water quality by comparison between hoshangabad (non industrial area) and mandideep (industrial area), Bhopal (India), IJRET: International Journal of Research in Engineering and Technology, 03 (12):144-146.

Moharir, A., D.S. Ramteke, C.A. Moghe, S.R. Wate and R. Sarin 2002,Surface and ground water quality assessment in Bina region. Ind. J. Environ. Protec., 22(9): 961-969.

Ramakrishnaiah, C. R, Sadashivaiah, C. and Ranganna, G., 2009. Assessment of Water Quality Index for the Groundwater in Tumkur Taluk,Karnataka State, India , CODEN ECJHAOE-Journal of Chemistryhttp, 6(2): 523530.

Sharma M.K., , and Jain C. K.., 2006. Multivariate analysis of groundwater quality data of District Jodhpur, Rajasthan, Journal of Environmental Science \& Engineering .48(4):271-280.

Sangu, R. P. S. and S. K. Sharma1987. An assessment of water quality of river Ganga at Garmukeshwar. Ind. $\boldsymbol{J}$. Ecol., 14(20):278-287.

Saxena Umesh and Saxena Swati 2015. Correlation study on physico-chemical parameters and qualityassessment of ground water of bassi tehsil of district jaipur, rajasthan, Suresh Gyan Vihar University International Journal of Environment, Science and Technology, 1 (1):78-91.

Shankar, B. S., Balasubramanya N. and Reddy M. T., 2007. Impact of industrialization on groundwater quality - a case study of Peenya industrial area, Bangalore, India, Environ Monit Assess , 142 (1-3):263-268.

Tyagi Mohit, Rai Swapnil, Kumar Ashwani,Yadav Ashwani, and Tyagi Hariom, 2015. Assessment of Ground Water Quality of Gajraula Industrial Area of Uttar Pradesh, International Journal of Scientific Research and Education,3(4):32653271.

Zaware Sandeep Gangadhar, Patil Vivekkumar V and Zaware Pushpa Sandeep 2015. Assessment of Ground Water Quality and its Impact on Human health At Padghe in Raigad District, Maharashtra, IndiaInternational Research Journal of Environment Sciences . 4(5): 57-61. 